\title{
Design, Synthesis, Pharmacological Evaluation and Molecular Docking Studies of Substituted Oxadiazolyl-2-Oxoindolinylidene Propane Hydrazide Derivatives
}

\author{
Deweshri Kerzare, ${ }^{a, b}$ Rupesh Chikhale, ${ }^{*, a, b, c}$ Ratnadeep Bansode, ${ }^{d}$ Nikhil Amnerkar, ${ }^{b}$ \\ Nazira Karodia, ${ }^{e}$ Anant Paradkar ${ }^{d}$ and Pramod Khedekar ${ }^{a}$ \\ ${ }^{a}$ Department of Pharmaceutical Sciences, Rashtrasant Tukadoji Maharaj Nagpur University, \\ Amravati Road, 440033 Nagpur-MS, India \\ ${ }^{b}$ Kamala Nehru College of Pharmacy, Butibori, 441108 Nagpur-MS, India \\ ${ }^{c}$ Advanced Centre for Treatment, Research and Education in Cancer (ACTREC), \\ Tata Memorial Centre, Kharghar, 410210 Navi Mumbai-MS, India \\ ${ }^{d}$ Centre for Pharmaceutical Engineering Science, Faculty of Life Sciences, University of Bradford, \\ BD7 1DP Bradford, United Kingdom \\ ${ }^{e}$ School of Biology, Chemistry and Forensic Science, University of Wolverhampton, \\ WV1 ILY Wolverhampton, United Kingdom
}

\begin{abstract}
The manuscript describes design and synthesis of novel oxadiazolyl-2-oxoindolinylidene propane hydrazides as amide tethered hybrids of indole and oxadiazole and their evaluation for antiinflammatory and analgesic activity. The compounds were synthesized following five step reaction to yield fifteen derivatives as 3-(5-substituted-1,3,4-oxadiazol-2-yl)- $\mathrm{N}^{\prime}$-[2-oxo-1,2-dihydro-3Hindol-3-ylidene]propane hydrazides. The final derivatives 3-[5-(4-hydroxyphenyl)-1,3,4-oxadiazol2-yl]- $N$ '-[2-oxo-1,2-dihydro-3H-indol-3-ylidene]propane hydrazide and 3-[5-(4-methylphenyl)1,3,4-oxadiazol-2-yl]- $N^{\prime}$-[2-oxo-1,2-dihydro-3H-indol-3-ylidene]propane hydrazide were found to be highly promising molecules with severity index of 0.35 and 0.56 , respectively, which is promising for an analgesic compound. The hydroxy and methyl substitution on phenyl ring system provided with active anti-inflammatory compounds having increase in reaction time of 84.11 and $83.17 \%$, respectively compared to standard drug at $85.84 \%$. Molecular docking studies exhibit comparable interaction with synthesized derivatives and standard drug having a dock score of -4.44 by the K-nearest neighbour genetic algorithm method.
\end{abstract}

Keywords: hybrid approach, indole, anti-inflammatory, analgesic, molecular docking

\section{Introduction}

Discovery of new molecules within a short possible time has become a focal point in current medicine. Need for new and better drugs with less toxicity and more selectivity are major criteria for the designing of a molecule. Recently, many new approaches are observed to be practiced for development of newer biologically active molecules. Special focus is given on structure based drug discovery, fragment based drug discovery, protein-protein interaction inhibitor study, proteomics and pharmacogenomics and these are becoming more and more popular to medicinal chemists. ${ }^{1}$ Still the earlier methods are persistent and yielding good

*e-mail: rupeshchikhale7@gmail.com results in form of newer and safer molecules. One of such approach is hybrid approach. ${ }^{2}$ It involves development of better, synergistic molecules on hybridization of two or more active biomolecules or ligands to develop newer derivative that possess good pharmacological activity. The success of this approach has been unequivocal with reports of development of anticancer hybrid agents, several nonsteroidal anti-inflammatory drugs (NSAIDs) and antiviral agents. Many drugs that are developed on basis of hybrid approach are used in therapy today and many more are in pipelines and yet to come on market.

The success of hybrid approach is depicted by several molecules such as cediranib, which is a hybrid of quinazoline and indole; ateviridine, a hybrid of pyridine and indole; indalpine, a hybrid of indole; and 


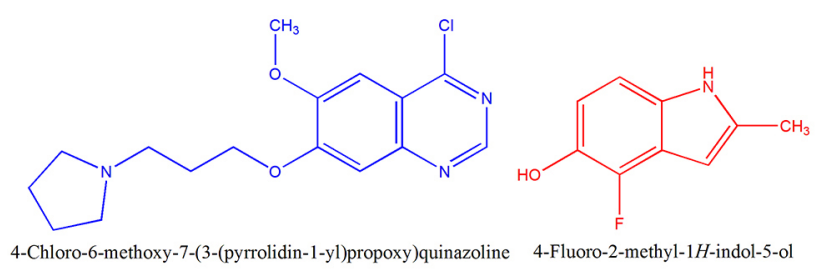

4-Chloro-6-methoxy-7-(3-(pyrrolidin-1-yl)propoxy)quinazoline 4-Fluoro-2-methyl-1H-indol-5-ol

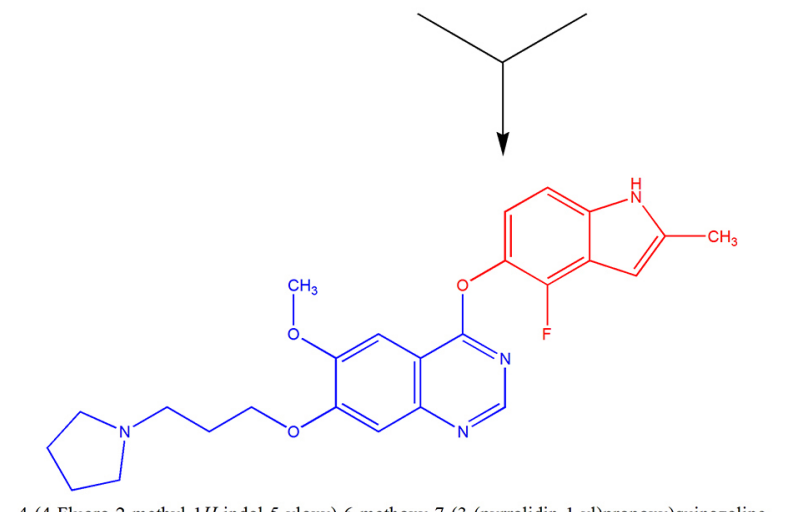

Drug name: Cediranib

Class: vascular endothelial growth factor (VEGF) inhibitor
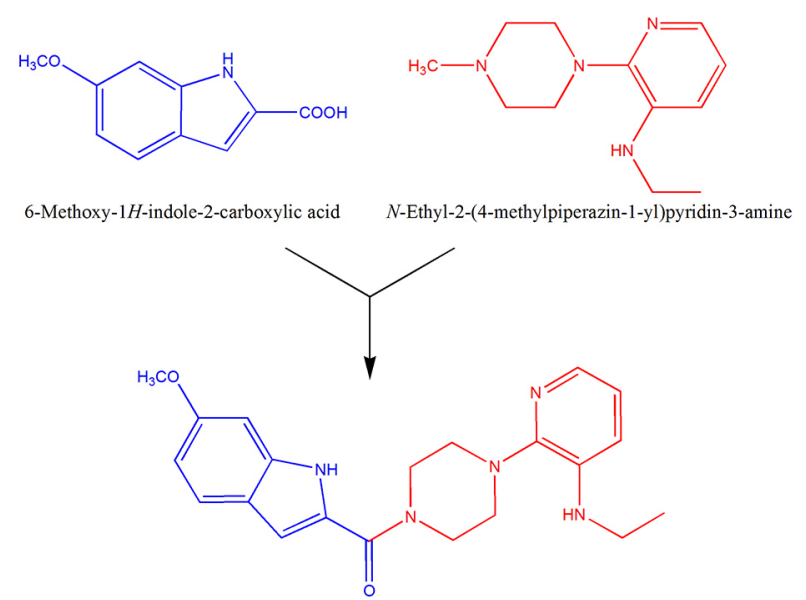

(4-(3-(Ethylamino)pyridin-2-yl)piperazin-1-yl)(6-methoxy-1H-indol-2-yl)methanone

Drug name: Ateviridine

Class: non-nucleoside reverse transcriptase inhibitor or the anti-HIV drug

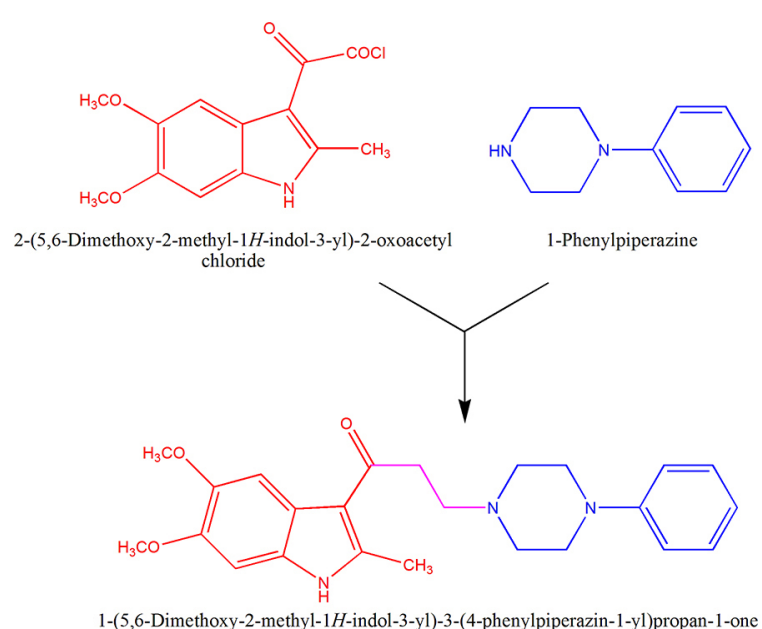

Drug name: Indalpine

Class: antipsychotic drug used to treat schizophrenia
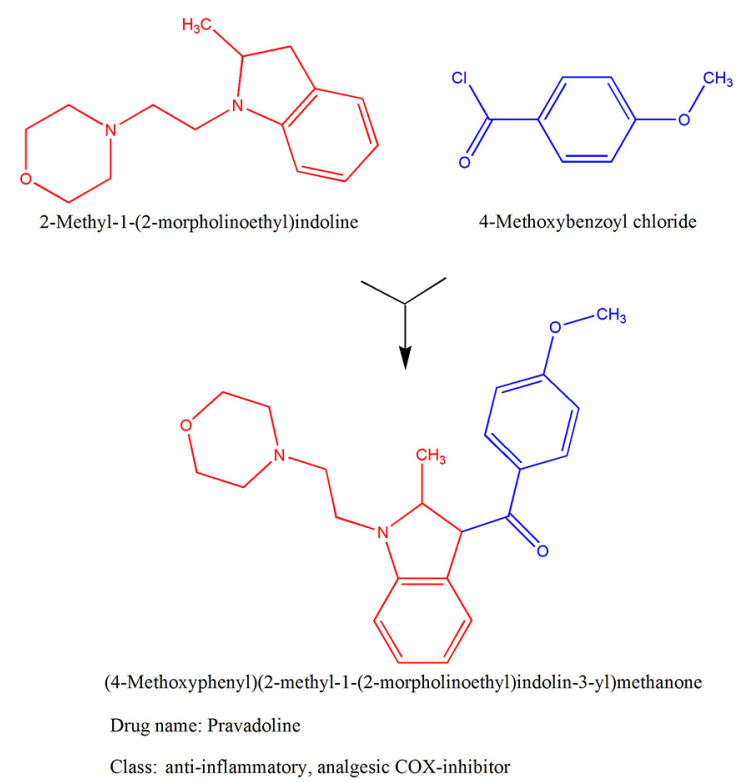

Figure 1. Examples describing success story of hybrid approach in drug discovery.

piperazine and pravadoline, which is a hybrid of indole and morpholine, respectively (Figure 1). The success of this approach is well defined in discovery of NSAIDs such as the pravadoline, which acts as cyclooxygenase (COX) inhibitor but also exhibit a potent cannabinoid agonist effect. ${ }^{3}$ The need for efficient and nontoxic NSAIDs has made us to design and develop newer and better molecules. The unwanted gastrointestinal (GI) ulceration, bleeding, nephrotoxicity observed in conventional COX inhibitors and the failure of selective COX inhibitors due to adverse cardiac effects suggest for better drugs devoid of these severe side effects. ${ }^{4}$ In view of this scenario, it was decided to employ the hybrid approach for development of novel molecules for their analgesic and anti-inflammatory activity. To achieve this goal, we selected to design and synthesize a hybrid molecule from indole and oxadiazole nuclei. Indole derivatives have been found to exhibit variety of pharmacological activities and have raised considerable interest because of their potential beneficial effects on human health. ${ }^{5,6}$ They have been reported to possess antibacterial, ${ }^{7}$ anticonvulsant, ${ }^{8}$ antifungal, ${ }^{9}$ antiviral, ${ }^{10}$ anticancer ${ }^{11}$ and anti-inflammatory ${ }^{12}$ properties. 
Indole derivative such as indomethacin is an established NSAID but it cause severe gastrointestinal side effect. The oxadiazole is found to poses versatile activity like the antiviral, anticancer and anticystic activity; compounds bearing 1,3,4-oxadiazole nucleus are also known to exhibit unique antiedema and anti-inflammatory potential. ${ }^{13}$ In the past, many indole and 1,3,4-oxadiazole derivatives showed potential for anti-inflammatory and analgesic activity in animal model of inflammation and pain. ${ }^{14-18}$

Step $(i)$<smiles>[R]C(=O)O</smiles>

Substituted benzoic acid

1-15<smiles>[R]C(=O)OC</smiles>

Susbtituted ethyl benzoates

16-30

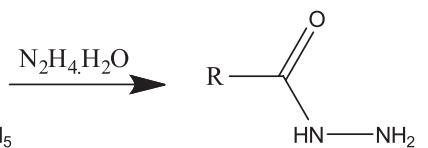

Substituted aryl acid hydrazides

31-45

Step (ii)<smiles>O=C1Nc2ccccc2C1=O</smiles>

Indoline-2,3-dione

46<smiles>N/N=C1\C(=O)Nc2ccccc21</smiles>

3-Hydrazonoeindolin-2-one 47<smiles>C/C=C\C=[Si](C)\C=C\C=C\C</smiles><smiles>O=C(O)CCC(=O)N/N=C1\C(=O)Nc2ccccc21</smiles>

3-(2-Oxoindolin-3-ylideneaminocarbamoyl)propanoic acid

48<smiles>[R]c1ccc(-c2nnc(CCC(=O)N/N=C3\C(=O)Nc4ccccc43)o2)cc1</smiles>

2-Oxoindolin-3-ylidene-3-(5-susbstituted phenyl-1,3,4-oxadiazol-2-yl)propanehydrazide

49-63

\begin{tabular}{|c|c|c|c|c|c|c|c|c|c|c|c|c|c|c|c|}
\hline Compound & 49 & 50 & 51 & 52 & 53 & 54 & 55 & 56 & 57 & 58 & 59 & 60 & 61 & 62 & 63 \\
\hline $\mathrm{R}$ & $\mathrm{H}$ & $p-\mathrm{OH}$ & $p-\mathrm{CH}_{3}$ & $m-\mathrm{NO}_{2}$ & $o-\mathrm{OCH}_{3}, m^{\prime}-\mathrm{OCH}_{3}$ & $p-\mathrm{Cl}$ & $p-\mathrm{NO}_{2}$ & $p-\mathrm{COCH}_{3}$ & $p-\mathrm{F}$ & $o-\mathrm{Cl}$ & $o-\mathrm{NO}_{2}$ & $m-\mathrm{Cl}$ & $o-\mathrm{F}$ & $p-\mathrm{Br}$ & $m-\mathrm{Br}$ \\
\hline
\end{tabular}

Scheme 1. Synthesis of $N^{\prime}$-(2-oxoindolin-3-ylidene)-3-(5-substituted phenyl-1,3,4-oxadiazol-2-yl)propane hydrazide derivatives 49-63. 
The need for newer drug molecule and success of hybrid approach compelled us for development of some new NSAIDs. The target moieties selected for formation of a hybrid drug was based on our previous literature study, and it was found that indole and oxadiazole nuclei possess analgesic and anti-inflammatory properties and thus it was found worth to prepare a hybrid derivative from these moieties. To achieve this goal, we synthesized fifteen different hybrids of indole and oxadiazole as 5 -substituted1,3,4-oxadiazole-2-yl-(2-oxo-1,2-dihydro-indol-3-ylidene) propane hydrazide derivatives. Scheme 1 explains the synthesis of these derivatives that involves two different steps. In the first step, substituted aryl acid hydrazides were obtained from various substituted benzoic acids with formation of intermediate benzoates. In the second step, the indole-2,3-dione was reacted with hydrazine hydrate to form isatin-3-hydrazone, which in the subsequent reaction with succinic anhydride produces indolyl hydrazinyl butonic acid. This acid on reaction with substituted aryl acid hydrazides obtained from earlier steps yields the final derivatives as substituted oxadiazolyl indolyl propane hydrazides.

These newly synthesized compounds were tested initially for their in vitro characteristic as active or inactive molecules by the egg albumin denaturation test. ${ }^{19}$ Further, these compounds were subjected for their analgesic and antiinflammatory activity by the carrageenan induced rat paw edema model ${ }^{20}$ and Eddy's hot plate method, ${ }^{21}$ respectively. After evaluation for pharmacological studies, it becomes necessary to determine the safety of synthesized compounds with respect to ulcerogenesis study or the determination of severity index with respect to reference drug such as indomethacin. Further, to determine the possible interaction of most potent compound and receptor in silico, studies need to be carried out. This was achieved by molecular docking studies of the ligands on the COX-II receptor. ${ }^{22,23}$

The series of final derivatives $N^{\prime}$-(2-oxoindolin-3ylidene)-3-(5-substituted phenyl-1,3,4-oxadiazol-2-yl) propane hydrazides 49-63 as described in this study is outlined in Scheme 1. The products were obtained following two different steps; in the first step various substituted benzoic acids 1-15 were obtained commercially; these substituents were reacted with ethanol in presence of sulphuric acid to derive corresponding benzoates 16-30 under conditions of nucleophilic substitution reaction. These benzoates were further treated with hydrazine hydrate to yield various substituted aryl acid hydrazides 3145, which were further employed in the reaction system. In the second step to the reaction scheme, isatin $\mathbf{4 6}$ was treated with hydrazine hydrate under ambient conditions to yield the isatin-3-hydrazone 47. It was further substituted by succinic anhydride to yield the oxoindolyidene hydrazinyl butanoic acid 48. Up to this moiety, the step two provides with reactant for the final reaction, in which the various substituted aryl acid hydrazides $\mathbf{3 1 - 4 5}$ and oxoindolyidene hydrazinyl butanoic acid $\mathbf{4 8}$ react to undergo the cyclization reaction in presence of phosphorous oxychloride, yielding various $N^{\prime}$-(2-oxoindolin-3-ylidene)-3-(5-substituted phenyl-1,3,4-oxadiazol-2-yl) propane hydrazides 49-63 in $40-80 \%$ yield.

\section{Experimental}

\section{Materials and methods}

Chemicals were obtained from Sigma-Aldrich (St. Louis, MO, USA), S D Fine-Chem (Mumbai, MH, India) and Merck (Darmstadt, Germany), unless specified. Melting points (m.p.) were detected with open capillaries using ThermoNik precision melting point cum boiling point apparatus (model C-PMB-2, Mumbai, MH, India) and are uncorrected. Infrared (IR) spectra (KBr) were recorded on FTIR-8400s spectrophotometer (Shimadzu, Tokyo, Japan) at the Department of Pharmaceutical Sciences, Rashtrasant Tukadoii Maharai (RTM) Nagpur University. Proton $\left({ }^{1} \mathrm{H}\right)$ and carbon $13\left({ }^{13} \mathrm{C}\right)$ nuclear magnetic resonance (NMR) were obtained using a Bruker Avance II $400 \mathrm{MHz}$ spectrometer (Billerica, MA, USA), using tetramethylsilane (TMS) as internal standard. All chemical shift values were recorded as $\delta$ (ppm), coupling constant value $J$ is measured in hertz, the peaks are presented as s (singlet), d (doublet), $\mathrm{t}$ (triplet), brs (broad singlet), dd (double doublet), $\mathrm{m}$ (multiplet). The purity of compounds was controlled by thin layer chromatography (silica gel HF254e361, type 60, $0.25 \mathrm{~mm}$; Merck, Darmstadt, Germany). Electrospray ionization mass spectrometry (ESI-MS) was recorded at Waters Q-TOF spectrometer (Waters, Milford, MA, USA) at the Sophisticated Analytical Instrumentation Facility (SAIF), Punjab University (Chandigarh, PB, India).

\section{Synthesis}

General methods for synthesis of substituted ethyl benzoates (16-30 $)^{24}$

To a solution of substituted benzoic acid (1-15) $(0.246 \mathrm{~mol})$ in dry ethanol $(2.5 \mathrm{~mol})$, concentrated sulphuric acid $(0.5 \mathrm{~mL})$ was added. The reaction mixture was refluxed for $8 \mathrm{~h}$. Excess of ethanol was distilled off and the content was allowed to cool. The residue was poured into separating funnel containing $60 \mathrm{~mL}$ of water. Carbon-tetrachloride (5-10 mL) was added to obtain sharp separation of aqueous and ester layer. Ester layer was washed with sodium hydrogen carbonate solution. The esters (16-30) were 
collected and recrystallized from ethanol. Details of these compounds are available in Supplementary Information.

General methods for synthesis of substituted aryl acid hydrazides (31-45) ${ }^{24}$

The substituted ethyl benzoates (16-30) (0.01 mol) dissolved in dry ethanol $(25 \mathrm{~mL})$, hydrazine hydrate $(99 \%$, $0.01 \mathrm{~mol}$ ) was added and the mixture was refluxed for $6 \mathrm{~h}$. The reaction mixture was cooled and the solid obtained was filtered and recrystallized from dilute ethanol or from water. Details of these compounds are available in Supplementary Information.

Procedure for synthesis of 3-hydrazinylidene-1,3-dihydro$2 \mathrm{H}$-indol-2-one $(\mathbf{4 7})^{25}$

A mixture of isatin (46) (1 mmol) and hydrazine hydrate (99\%, $0.055 \mathrm{~g}, 1.1 \mathrm{mmol})$ in absolute methanol (25 mL) was refluxed for $1 \mathrm{~h}$ and then cooled to room temperature. The precipitate of hydrazones was filtered and dried. The crude product was recrystallized from ethanol to give hydrazones (47).

Yield: $1.0 \mathrm{~g}(70 \%)$; m.p. $248-250{ }^{\circ} \mathrm{C} ; \mathrm{R}_{\mathrm{f}} 0.39$ (methanol:toluene, 1:4); IR (KBr) $v / \mathrm{cm}^{-1} 3411,2916$, 1655, 1618; ${ }^{1} \mathrm{H}$ NMR (DMSO) $\delta 7.63$ (s, 1H, Ar-H), 7.45 (s, 1H, Ar-H), 7.31 (s, 1H, Ar-H), 7.20 (s, 1H, Ar-H), 7.0 (s, 1H, NH), 3.34 (s, 2H, NH); ${ }^{13} \mathrm{C} \mathrm{NMR} \mathrm{(100} \mathrm{MHz,}$ DMSO- $\left.d_{6}\right) \delta 166.25,152.64,130.73,125.24,120.75$, 120.61, 117.55, 78.81; EI-MS m/z [M + H $]^{+} 162.24$.

Procedure for synthesis of 4-oxo-4-[2-(2-oxo-1,2-dihydro$3 \mathrm{H}$-indol-3-ylidene)-hydrazinyl]butanoic acid $(\mathbf{4 8})^{26}$

The mixture of compound (47) $(0.01 \mathrm{~mol})$, succinic anhydride $(0.01 \mathrm{~mol})$ and trimethylamine $(1 \mathrm{mmol})$ in dichloromethane (DCM) was stirred at room temperature for $3 \mathrm{~h}$ and diluted with DCM. The solution was washed with sodium bicarbonate and brine twice. The product thus obtained was dried over sodium sulphate and the excess of solvent was distilled off. The resulting solid was recrystallized from ethanol.

Yield: 0.08 g (80\%); m.p. $238-240{ }^{\circ} \mathrm{C} ; \mathrm{R}_{\mathrm{f}} 0.58$ (toluene); IR (KBr) v / cm ${ }^{-1} 3394,3059,2916,1655,1618 ;{ }^{1} \mathrm{H}$ NMR (DMSO) $\delta 12.44$ (s, 1H, OH), 7.99 (t, 1H, J 6.7 Hz, Ar-H), 7.97 (s, 1H, Ar-H), 7.44 (d, 1H, J $8.2 \mathrm{~Hz}, \mathrm{Ar}-\mathrm{H}), 7.31$ (d, 1H, J7.2 Hz, Ar-H), 6.30 (s, 1H, NH), 3.78 (s, 2H, $\mathrm{CH}_{2}$ ), $2.23\left(\mathrm{~s}, 2 \mathrm{H}, \mathrm{CH}_{2}\right) ;{ }^{13} \mathrm{C}$ NMR $\left(100 \mathrm{MHz}, \mathrm{DMSO}-d_{6}\right) \delta 166.22$, $157.59,148.41,131.35,126.12,123.58,121.71,120.58$, 118.78, 106.41, 51.01, 30.31; EI-MS m/z [M + H] 262.04 .

General procedure for synthesis of 3-(5-substituted-1,3,4oxadiazol-2-yl)-N'-[2-oxo-1,2-dihydro-3H-indol-3-ylidene] propane hydrazide derivatives $(49-63)^{27}$

To a solution of compound (31-45) $(0.01 \mathrm{~mol})$ in phosphorous oxychloride (15-20 mL), compound (48) $(0.01 \mathrm{~mol})$ was added. The reaction mixture was refluxed for $5 \mathrm{~h}$. The mixture was cooled to room temperature and poured onto crushed ice and neutralized the contents with sodium hydroxide solution $\left(1 \mathrm{~mol} \mathrm{~L}^{-1}\right)$. The product was filtered, washed with water, dried and recrystallized using methanol to get 49-63.

3-(5-Phenyl-1,3,4-oxadiazol-2-yl)-N'-[2-oxo-1,2-dihydro-3Hindol-3-ylidene]propane hydrazide (49)

Yield: $2.03 \mathrm{~g}$ (78\%); m.p. $208-210^{\circ} \mathrm{C} ; \mathrm{R}_{\mathrm{f}} 0.83$ (chloroform); $\lambda_{\max } 448 \mathrm{~nm}$; IR (KBr) $v / \mathrm{cm}^{-1} 3254$, 3059, 2916, 1655, 1618; ${ }^{1} \mathrm{H}$ NMR (DMSO) $\delta 8.91$ (s, 1H, Ar-H), 8.59 (s, 1H, Ar-H), 8.0 (s, 1H, Ar-H), 7.98 (d, 2H, J 7.9 Hz, Ar-H), 7.76 (t, 2H, J 8.3 Hz, Ar-H), 7.45 (dd, 1H, $J 7.4 \mathrm{~Hz}, \mathrm{Ar}-\mathrm{H}), 7.32$ (dd, 1H, J7.6 Hz, Ar-H), 3.58 (s, 2H, $\left.\mathrm{CH}_{2}\right), 2.38\left(\mathrm{~s}, 2 \mathrm{H}, \mathrm{CH}_{2}\right) ;{ }^{13} \mathrm{C}$ NMR (100 MHz, DMSO- $\left.d_{6}\right)$ $\delta 160.53,156.17,148.33,131.52,126.20,123.76,121.78$, 120.72, 33.98; EI-MS $\mathrm{m} / z[\mathrm{M}+\mathrm{H}]^{+}$362.58; anal. calcd. for $\mathrm{C}_{19} \mathrm{H}_{15} \mathrm{~N}_{5} \mathrm{O}_{3}: \mathrm{C}, 63.1 ; \mathrm{H}, 4.15 ; \mathrm{N}, 19.37$; found: C, 63.3; H, 4.17; N, 19.39.

3-[5-(4-Hydroxyphenyl)-1,3,4-oxadiazol-2-yl]-N'-[2-oxo-1,2dihydro-3H-indol-3-ylidene]propane hydrazide (50)

Yield: 2.08 g (80\%), m.p. $210-212^{\circ} \mathrm{C} ; \mathrm{R}_{\mathrm{f}} 0.8$ (chloroform); $\lambda_{\max } 442 \mathrm{~nm}$; IR (KBr) v / cm ${ }^{-1} 3585,3211$, 2849, 2978, 1711, 1605; ${ }^{1} \mathrm{H}$ NMR (DMSO) $\delta 9.49$ (s, 1H, $\mathrm{OH}), 7.72$ (t, 2H, J $6.9 \mathrm{~Hz}, \mathrm{Ar}-\mathrm{H}), 7.40$ (t, 2H, J 8.1 Hz, Ar-H), 7.21 (t, 1H, J 6.9 Hz, Ar-H), 7.18 (d, 1H, J 7.4 Hz, Ar-H), 6.65 (t, 1H, J 7.8 Hz, Ar-H), 6.36 (s, 1H, Ar-H), 2.31 (s, 2H, $\left.\mathrm{CH}_{2}\right), 1.23$ (s, 2H, $\left.\mathrm{CH}_{2}\right) ;{ }^{13} \mathrm{C} \mathrm{NMR}(100 \mathrm{MHz}$, DMSO- $\left.d_{6}\right) \delta 166.11,162.44,157.29,153.76,137.59$, $132.14,128.25,126.72,122.81,115.18,112.36,102.89$, 50.84, 23.17; EI-MS m/z [M + H] 378.11; anal. calcd. for $\mathrm{C}_{19} \mathrm{H}_{15} \mathrm{~N}_{5} \mathrm{O}_{4}$ : C, 63.4; H, 4.13; N, 19.33; found: C, 63.6; H, 4.17; N, 19.35 .

3-[5-(4-Methylphenyl)-1,3,4-oxadiazol-2-yl]- $N$-[2-oxo-1,2dihydro-3H-indol-3-ylidene]propane hydrazide (51)

Yield: $1.82 \mathrm{~g}$ (70\%); m.p. 200-202 ${ }^{\circ} \mathrm{C} ; \mathrm{R}_{\mathrm{f}} 0.47$ (chloroform); $\lambda_{\max } 357 \mathrm{~nm}$; IR (KBr) $v / \mathrm{cm}^{-1} 3249,2849$, 2918, 1614, 1598; ${ }^{1} \mathrm{H}$ NMR (DMSO) $\delta 7.75$ (t, $1 \mathrm{H}, J 8.1 \mathrm{~Hz}$, Ar-H), 7.46 (t, 1H, J 7.6 Hz, Ar-H), 7.34 (t, 1H, J 5.9 Hz, Ar-H), 7.19 (d, 1H, J 7.8 Hz, Ar-H), 7.02 (m, 2H, J 7.9 Hz, Ar-H), 6.82 (t, 1H, J $8.4 \mathrm{~Hz}, \mathrm{Ar}-\mathrm{H}), 6.43$ (s, 1H, Ar-H), 3.34 (s, 4H, $\mathrm{CH}_{2}$ ), 2.32 (s, 3H, $\left.\mathrm{CH}_{3}\right) ;{ }^{13} \mathrm{C} \mathrm{NMR}(100 \mathrm{MHz}$, DMSO- $\left.d_{6}\right) \delta 166.37,166.05,162.55,158.97,153.97,152.79$, $137.52,133.74,130.88,128.22,126.76,122.81,120.82$, $117.68,113,56,112.36,102.79,55.90,54.94,23.20$; EI-MS $m / z[\mathrm{M}+\mathrm{H}]^{+}$376.31; anal. calcd. for $\mathrm{C}_{20} \mathrm{H}_{17} \mathrm{~N}_{5} \mathrm{O}_{3}: \mathrm{C}, 64.1$; H, 4.6; N, 18.7; found: C, 64.6; H, 4.8; N, 18.91 . 
3-[5-(3-Nitrophenyl)-1,3,4-oxadiazol-2-yl]- $N$-[2-oxo-1,2dihydro-3H-indol-3-ylidene]propane hydrazide (52)

Yield: $1.87 \mathrm{~g}(72 \%) ;$ m.p. $248-250{ }^{\circ} \mathrm{C} ; \mathrm{R}_{\mathrm{f}} 0.72$ (chloroform); $\lambda_{\max } 447 \mathrm{~nm}$; IR (KBr) $v / \mathrm{cm}^{-1} 3084,2918$, $1655,1618,1529 ;{ }^{1} \mathrm{H}$ NMR (DMSO) $\delta 8.31$ (s, $1 \mathrm{H}, \mathrm{Ar}-\mathrm{H}$ ), 7.87 (d, 2H, J 7.3 Hz, Ar-H), 7.61 (t, 2H, J 8.4 Hz, Ar-H), $7.21(\mathrm{t}, 2 \mathrm{H}, J 7.9 \mathrm{~Hz}, \mathrm{Ar}-\mathrm{H}), 6.62(\mathrm{~s}, 1 \mathrm{H}, \mathrm{Ar}-\mathrm{H}), 3.63$ (s, $\left.2 \mathrm{H}, \mathrm{CH}_{2}\right), 2.34\left(\mathrm{~s}, 2 \mathrm{H}, \mathrm{CH}_{2}\right) ;{ }^{13} \mathrm{C}$ NMR $(100 \mathrm{MHz}$, DMSO- $\left.d_{6}\right) \delta 165.75,163.11,155.20,147.82,143.43$, $137.15,133.34,130.43,127.01,124.34,123.33,123.02$, 122.80, 121.50, 112.37, 101.87, 51.03, 23.38; EI-MS m/z $[\mathrm{M}+\mathrm{H}]^{+}$407.72; anal. calcd. for $\mathrm{C}_{19} \mathrm{H}_{14} \mathrm{~N}_{6} \mathrm{O}_{5}: \mathrm{C}, 56.3 ; \mathrm{H}$, $3.5 ; \mathrm{N}, 20.77$; found: $\mathrm{C}, 56.5 ; \mathrm{H}, 3.8 ; \mathrm{N}, 20.79$.

3-[5-(2,3-Dimethoxyphenyl)-1,3,4-oxadiazol-2-yl]- $N$-[2oxo-1,2-dihydro-3H-indol-3-ylidene]propane hydrazide (53)

Yield: 2.16 g (83\%); m.p. $230-232{ }^{\circ} \mathrm{C} ; \mathrm{R}_{\mathrm{f}} 0.69$ (chloroform); $\lambda_{\max } 325 \mathrm{~nm}$; IR (KBr) $v / \mathrm{cm}^{-1} 3293,2918$, $1655,1618,2835,1425 ;{ }^{1} \mathrm{H}$ NMR (DMSO) $\delta 7.73$ (s, $1 \mathrm{H}$, Ar-H), 7.21 (d, 2H, J 8.4 Hz, Ar-H), 7.19 (s, 1H, Ar-H), 7.05 (s, 1H, Ar-H), 6.76 (t, 1H, J 7.9 Hz, Ar-H), 6.66 (s, $1 \mathrm{H}, \mathrm{Ar}-\mathrm{H}), 4.07$ (t, $\left.2 \mathrm{H}, J 7.9 \mathrm{~Hz}, \mathrm{CH}_{2}\right), 3.34(\mathrm{t}, 2 \mathrm{H}$, $\left.J 8.4 \mathrm{~Hz}, \mathrm{CH}_{2}\right), 2.50\left(\mathrm{~s}, 3 \mathrm{H}, \mathrm{O}-\mathrm{CH}_{3}\right), 1.22(\mathrm{~d}, 3 \mathrm{H}, J 7.8 \mathrm{~Hz}$, $\left.\mathrm{O}-\mathrm{CH}_{3}\right) ;{ }^{13} \mathrm{C}$ NMR (100 MHz, DMSO- $\left.d_{6}\right) \delta 165.64$, 162..36, 153.50, 147.05, 146.48, 137.67, 132.76, 126.71, 122.80, 119.49, 115.51, 112.52, 111.62, 102.97, 56.38, 55.57, 23.08, 14.13; EI-MS $m / z[\mathrm{M}+\mathrm{H}]^{+}$422.14; anal. calcd. for $\mathrm{C}_{21} \mathrm{H}_{19} \mathrm{~N}_{5} \mathrm{O}_{5}: \mathrm{C}, 59.9 ; \mathrm{H}, 4.52 ; \mathrm{N}, 16.65$; found: C, $59.5 ; \mathrm{H}, 4.8 ; \mathrm{N}, 16.69$.

3-[5-(4-Chlorophenyl)-1,3,4-oxadiazol-2-yl]- $N$-[2-oxo-1,2dihydro-3H-indol-3-ylidene]propane hydrazide (54)

Yield: 1.12 g (43\%); m.p. $240-244{ }^{\circ} \mathrm{C} ; \mathrm{R}_{\mathrm{f}} 0.66$ (chloroform); $\lambda_{\max } 452 \mathrm{~nm}$; IR (KBr) $v / \mathrm{cm}^{-1} 3239,2843$, $1713,1547,2902 ;{ }^{1} \mathrm{H}$ NMR (DMSO) $\delta 8.98$ (s, $1 \mathrm{H}, \mathrm{Ar}-\mathrm{H}$ ), 8.59 (s, 1H, Ar-H), 8.0 (d, 2H, J 8.1 Hz, Ar-H), 7.75 (t, $2 \mathrm{H}, J 7.9 \mathrm{~Hz}, \mathrm{Ar}-\mathrm{H}), 7.45$ (dd, $1 \mathrm{H}, J 7.4 \mathrm{~Hz}, \mathrm{Ar}-\mathrm{H}), 7.32$ (dd, $1 \mathrm{H}, J 7.9 \mathrm{~Hz}, \mathrm{Ar}-\mathrm{H}), 3.50$ (s, 2H, $\left.\mathrm{CH}_{2}\right), 2.31$ (s, $2 \mathrm{H}$, $\left.\mathrm{CH}_{2}\right) ;{ }^{13} \mathrm{C}$ NMR $\left(100 \mathrm{MHz}\right.$, DMSO- $\left.d_{6}\right) \delta 160.56,156.15$, 148.33, 131.50, 126.72, 126.21, 123.77, 121.79, 120.72, 115.25, 51.01, 33.92; EI-MS m/z [M + H]+ 397.32; anal. calcd. for $\mathrm{C}_{19} \mathrm{H}_{14} \mathrm{ClN}_{5} \mathrm{O}_{3}: \mathrm{C}, 57.62 ; \mathrm{H}, 3.58 ; \mathrm{N}, 17.71$; found: C, $57.68 ; \mathrm{H}, 3.62 ; \mathrm{N}, 18.1$.

3-[5-(4-Nitrophenyl)-1,3,4-oxadiazol-2-yl]- $N$-[2-oxo-1,2dihydro-3H-indol-3-ylidene]propane hydrazide (55)

Yield: 2.32 g (89\%); m.p. $240-244{ }^{\circ} \mathrm{C} ; \mathrm{R}_{\mathrm{f}} 0.71$ (chloroform); $\lambda_{\max } 448 \mathrm{~nm}$; IR (KBr) $v / \mathrm{cm}^{-1} 3121,2916$, $1655,1618,2849,1522$; ${ }^{1} \mathrm{H}$ NMR (DMSO) $\delta 8.15$ (s, $1 \mathrm{H}, \mathrm{Ar}-\mathrm{H}), 7.74$ (q, 1H, J $7.9 \mathrm{~Hz}, \mathrm{Ar}-\mathrm{H}), 7.46(\mathrm{t}, 1 \mathrm{H}$, $J 7.5 \mathrm{~Hz}, \mathrm{Ar}-\mathrm{H}), 7.31(\mathrm{dd}, 2 \mathrm{H}, J 8.2 \mathrm{~Hz}, \mathrm{Ar}-\mathrm{H}), 7.21$ (dd, 2H, J $8.4 \mathrm{~Hz}, \mathrm{Ar}-\mathrm{H}), 6.68$ (s, 1H, Ar-H), 3.35 (s, 2H, $\left.\mathrm{CH}_{2}\right), 2.51\left(\mathrm{~s}, 2 \mathrm{H}, \mathrm{CH}_{2}\right) ;{ }^{13} \mathrm{C}$ NMR (100 MHz, DMSO- $d_{6}$ ) $\delta$ 165.71, 163.07, 155.16, 149.14, 147.20, 137.16, 128.28, 126.96, 123.96, 122.80, 112.24, 101.69, 55.87, 23.37; EI-MS $m / z[\mathrm{M}+\mathrm{H}]^{+} 407.16$; anal. calcd. for $\mathrm{C}_{19} \mathrm{H}_{14} \mathrm{~N}_{6} \mathrm{O}_{5}$ : C, 56.3; H, 3.48; N, 20.68; found: C, 56.6; H, 3.5; N, 20.69.

3-[5-(4-Acetylphenyl)-1,3,4-oxadiazol-2-yl]- $N$-[2-oxo-1,2dihydro-3H-indol-3-ylidene]propane hydrazide (56)

Yield: $2.08 \mathrm{~g}$ (80\%); m.p. $166-170{ }^{\circ} \mathrm{C}, \mathrm{R}_{\mathrm{f}} 0.65$ (chloroform); $\lambda_{\max } 525 \mathrm{~nm}$; IR (KBr) v / cm ${ }^{-1} 3107$, 2918, 1648, 1603, 1210, 2849; ${ }^{1} \mathrm{H}$ NMR (DMSO): $\delta 7.73$ (t, $1 \mathrm{H}, J 7.4 \mathrm{~Hz}, \mathrm{Ar}-\mathrm{H}), 7.49$ (d, 1H, J 7.9 Hz, Ar-H), 7.19 (t, 2H, J 8.5 Hz, Ar-H), 7.03 (d, 1H, J 8.3 Hz, Ar-H), 6.75 (d, $1 \mathrm{H}, J 7.4 \mathrm{~Hz}, \mathrm{Ar}-\mathrm{H}), 6.65$ (s, 1H, Ar-H), 6.37 (s, 1H, $\mathrm{Ar}-\mathrm{H}$ ), 3.71 (s, $2 \mathrm{H}, \mathrm{CH}_{2}$ ), 3.35 (s, $2 \mathrm{H}, \mathrm{CH}_{2}$ ), 2.50 (s, 3H, $\left.\mathrm{CH}_{3}\right) ;{ }^{13} \mathrm{C}$ NMR $\left(100 \mathrm{MHz}\right.$, DMSO- $\left.d_{6}\right) \delta 200.86,166.12$, $162.48,153.70,147.14,146.49,137.65,132.70,126.74$, $123.93,122.80,119.31,115.50,112.50,111.41,102.84$, 56.29, 55.58, 50.82, 23.13; EI-MS $m / z[\mathrm{M}+\mathrm{H}]^{+}$404.09; anal. calcd. for $\mathrm{C}_{21} \mathrm{H}_{17} \mathrm{~N}_{5} \mathrm{O}_{4}: \mathrm{C}, 62.41 ; \mathrm{H}, 4.23 ; \mathrm{N}, 17.39$; found: C, $62.48 ; \mathrm{H}, 4.28 ; \mathrm{N}, 17.42$.

3-[5-(4-Fluorophenyl)-1,3,4-oxadiazol-2-yl]- $N$-[2-oxo-1,2dihydro-3H-indol-3-ylidene]propane hydrazide (57)

Yield: $2.14 \mathrm{~g}(82 \%)$; m.p. $182-184{ }^{\circ} \mathrm{C}, \mathrm{R}_{\mathrm{f}} 0.65$ (chloroform), $\lambda_{\max } 398 \mathrm{~nm}$, IR (KBr) $v / \mathrm{cm}^{-1} 2992$, 2916, 1655, 1605, 1097; ${ }^{1} \mathrm{H}$ NMR (DMSO) $\delta 8.10$ (d, 2H, $J 8.3 \mathrm{~Hz}, \mathrm{Ar}-\mathrm{H}), 7.68$ (t, 2H, J 7.6 Hz, Ar-H), 7.26 (dd, 1H, J7.6 Hz, Ar-H), 7.24 (dd, 1H, J7.3 Hz, Ar-H), 6.81 (t, $2 \mathrm{H}, J 6.9 \mathrm{~Hz}, \mathrm{Ar}-\mathrm{H}), 3.73\left(\mathrm{~s}, 2 \mathrm{H}, \mathrm{CH}_{2}\right), 2.50(\mathrm{~s}, 2 \mathrm{H}$, $\left.\mathrm{CH}_{2}\right) ;{ }^{13} \mathrm{C}$ NMR $\left(100 \mathrm{MHz}, \mathrm{DMSO}-d_{6}\right) \delta 164.52,163.31$, $161.31,160.36,135.94,129.96,129.88,127.96,124.35$, 124.17, 115.94, 115.72, 114.50, 103.80, 99.49, 57.01, 51.68; EI-MS $m / z,[\mathrm{M}+\mathrm{H}]^{+}$380.12; anal. calcd. for $\mathrm{C}_{19} \mathrm{H}_{14} \mathrm{FN}_{5} \mathrm{O}_{3}: \mathrm{C}, 60.31 ; \mathrm{H}, 3.71 ; \mathrm{N}, 18.48$; found: $\mathrm{C}, 60.38$; $\mathrm{H}, 3.78 ; \mathrm{N}, 18.52$.

3-[5-(2-Chlorophenyl)-1,3,4-oxadiazol-2-yl]- $N$-[2-oxo-1,2dihydro-3H-indol-3-ylidene]propane hydrazide (58)

Yield: 1.15 g (43\%); m.p. $244-246{ }^{\circ} \mathrm{C} ; \mathrm{R}_{\mathrm{f}} 0.61$ (chloroform); $\lambda_{\max } 455 \mathrm{~nm}$; IR (KBr) $v / \mathrm{cm}^{-1} 3235,2843$, 1713, 1537, 2902, 725; ${ }^{1} \mathrm{H}$ NMR (DMSO) $\delta 8.76$ (s, $1 \mathrm{H}$, $\mathrm{Ar}-\mathrm{H}), 8.31$ (s, 1H, Ar-H), 7.92 (d, 2H, J 8.4 Hz, Ar-H), $7.70(\mathrm{t}, 2 \mathrm{H}, J 7.6 \mathrm{~Hz}, \mathrm{Ar}-\mathrm{H}), 7.43$ (dd, $1 \mathrm{H}, J 7.5 \mathrm{~Hz}, \mathrm{Ar}-\mathrm{H}$ ), 7.30 (dd, $1 \mathrm{H}, J 7.8 \mathrm{~Hz}, \mathrm{Ar}-\mathrm{H}), 3.40\left(\mathrm{~s}, 2 \mathrm{H}, \mathrm{CH}_{2}\right), 2.53$ (s, $\left.2 \mathrm{H}, \mathrm{CH}_{2}\right) ;{ }^{13} \mathrm{C}$ NMR (100 MHz, DMSO- $d_{6}$ ) $\delta 164.16$, 154.17, 149.38, 133.60, 125.12, 125.26, 123.77, 121.80, 121.76, 116.27, 55.10, 34.82; EI-MS $m / z$ [M + H] $]^{+} 397.43$; anal. calcd. for $\mathrm{C}_{19} \mathrm{H}_{14} \mathrm{ClN}_{5} \mathrm{O}_{3}: \mathrm{C}, 57.62 ; \mathrm{H}, 3.58 ; \mathrm{N}, 17.71$; found: $\mathrm{C}, 57.68 ; \mathrm{H}, 3.62 ; \mathrm{N}, 18.1$. 
3-[5-(2-Nitrophenyl)-1,3,4-oxadiazol-2-yl]- $N$-[2-oxo-1,2dihydro-3H-indol-3-ylidene]propane hydrazide (59)

Yield: $2.22 \mathrm{~g}(89 \%) ;$ m.p. $242-244{ }^{\circ} \mathrm{C} ; \mathrm{R}_{\mathrm{f}} 0.71$ (chloroform); $\lambda_{\max } 438 \mathrm{~nm}$; IR (KBr) $v / \mathrm{cm}^{-1} 3121,2916$, $1645,1615,2749,1522 ;{ }^{1} \mathrm{H}$ NMR (DMSO) $\delta 8.31$ (s, $1 \mathrm{H}$, $\mathrm{Ar}-\mathrm{H}), 7.87(\mathrm{~d}, 1 \mathrm{H}, J 7.6 \mathrm{~Hz}, \mathrm{Ar}-\mathrm{H}), 7.78(\mathrm{~d}, 1 \mathrm{H}, J 7.4 \mathrm{~Hz}$, $\mathrm{Ar}-\mathrm{H}), 7.61(\mathrm{dd}, 2 \mathrm{H}, J 5.3 \mathrm{~Hz}, \mathrm{Ar}-\mathrm{H}), 7.21(\mathrm{t}, 2 \mathrm{H}, J 7.4 \mathrm{~Hz}$, $\mathrm{Ar}-\mathrm{H}), 6.72(\mathrm{t}, 1 \mathrm{H}, J 7.1 \mathrm{~Hz}, \mathrm{Ar}-\mathrm{H}), 3.63\left(\mathrm{~s}, 2 \mathrm{H}, \mathrm{CH}_{2}\right), 2.34$ (s, $\left.2 \mathrm{H}, \mathrm{CH}_{2}\right) ;{ }^{13} \mathrm{C}$ NMR $\left(100 \mathrm{MHz}\right.$, DMSO- $\left.d_{6}\right) \delta 165.75$, $163.11,155.20,147.82,143.43,137.15,133.34,130.43$, $127.01,124.34,123.33,123.02,122.80,121.50,112.37$ 101.87, 51.11, 23.38; EI-MS $m / z[\mathrm{M}+\mathrm{H}]^{+} 407.4$; anal. calcd. for $\mathrm{C}_{19} \mathrm{H}_{14} \mathrm{~N}_{6} \mathrm{O}_{5}: \mathrm{C}, 56.3 ; \mathrm{H}, 3.48 ; \mathrm{N}, 20.68$; found: C, 56.6; H, 3.5; N, 20.69 .

3-[5-(3-Chlorophenyl)-1,3,4-oxadiazol-2-yl]-N'-[2-oxo-1,2dihydro-3H-indol-3-ylidene]propane hydrazide (60)

Yield: $1.1 \mathrm{~g}(43 \%)$; m.p. $235-237{ }^{\circ} \mathrm{C} ; \mathrm{R}_{\mathrm{f}} 0.66$ (chloroform); $\lambda_{\max } 432 \mathrm{~nm}$; IR (KBr) $v / \mathrm{cm}^{-1} 3229,2743$, $1713,1538,2902,725 ;{ }^{1} \mathrm{H}$ NMR (DMSO) $\delta 8.56$ (s, $1 \mathrm{H}$, $\mathrm{Ar}-\mathrm{H}), 8.14$ (s, 1H, Ar-H), 7.87 (d, 2H, J 8.4 Hz, Ar-H), 7.65 (t, 2H, J 7.6 Hz, Ar-H), 7.45 (dd, $1 \mathrm{H}, J 7.5 \mathrm{~Hz}, \mathrm{Ar}-\mathrm{H})$, 7.25 (dd, $1 \mathrm{H}, J 7.8 \mathrm{~Hz}, \mathrm{Ar}-\mathrm{H}), 3.35$ (s, $\left.2 \mathrm{H}, \mathrm{CH}_{2}\right), 2.80$ (s, $\left.2 \mathrm{H}, \mathrm{CH}_{2}\right) ;{ }^{13} \mathrm{C}$ NMR (100 MHz, DMSO- $\left.d_{6}\right) \delta 172.16$, $159.17,144.48,136.60,125.16,123.26,123.17,121.85$, 121.70, 117.27, 56.10, 33.83; EI-MS $m / z[\mathrm{M}+\mathrm{H}]^{+} 397.02$; anal. calcd. for $\mathrm{C}_{19} \mathrm{H}_{14} \mathrm{ClN}_{5} \mathrm{O}_{3}: \mathrm{C}, 57.62 ; \mathrm{H}, 3.58 ; \mathrm{N}, 17.71$; found: $\mathrm{C}, 57.68 ; \mathrm{H}, 3.62 ; \mathrm{N}, 18.1$.

3-[5-(2-Fluorophenyl)-1,3,4-oxadiazol-2-yl]- $N$-[2-oxo-1,2dihydro-3H-indol-3-ylidene]propane hydrazide (61)

Yield: $2.11 \mathrm{~g}(82 \%)$; m.p. $180-182{ }^{\circ} \mathrm{C}, \mathrm{R}_{\mathrm{f}} 0.65$ (chloroform), $\lambda_{\max } 368 \mathrm{~nm}$, IR (KBr) $v / \mathrm{cm}^{-1} 3297$, 2916, 1655, 1605, 1097; ${ }^{1} \mathrm{H}$ NMR (DMSO) $\delta 8.10$ (d, 2H, J 8.3 Hz, Ar-H), 7.68 (t, 2H, J 7.6 Hz, Ar-H), 7.26 (dd, $1 \mathrm{H}, J 7.6 \mathrm{~Hz}, \mathrm{Ar}-\mathrm{H}$ ), 7.24 (dd, 1H, J 7.3 Hz, Ar-H), 6.81 (t, $2 \mathrm{H}, J 6.9 \mathrm{~Hz}, \mathrm{Ar}-\mathrm{H}), 3.73$ (s, $\left.2 \mathrm{H}, \mathrm{CH}_{2}\right), 2.50$ (s, 2H, $\mathrm{CH}_{2}$ ); ${ }^{13} \mathrm{C}$ NMR (100 MHz, DMSO- $\left.d_{6}\right) \delta 164.52,163.31,161.31$, 160.36, 135.94, 129.96, 129.88, 127.96, 124.35, 124.17, 115.94, 115.72, 114.50, 103.80, 99.49, 57.01, 51.68; EI-MS $\mathrm{m} / z[\mathrm{M}+\mathrm{H}]^{+}$380.16; anal. calcd. for $\mathrm{C}_{19} \mathrm{H}_{14} \mathrm{FN}_{5} \mathrm{O}_{3}: \mathrm{C}, 60.31$; H, 3.71; N, 18.48; found: C, 60.38; H, 3.78; N, 18.52 .

3-[5-(4-Bromophenyl)-1,3,4-oxadiazol-2-yl]- $N$-[2-oxo-1,2dihydro-3H-indol-3-ylidene]propane hydrazide (62)

Yield: $2.64 \mathrm{~g}(82 \%) ;$ m.p. $174-176{ }^{\circ} \mathrm{C}, \mathrm{R}_{\mathrm{f}} 0.65$ (chloroform), $\lambda_{\max } 398 \mathrm{~nm}$, IR (KBr) v / cm ${ }^{-1}$ 2982, 2906, 1655,$1685 ;{ }^{1} \mathrm{H}$ NMR (DMSO) $\delta 8.91$ (s, $1 \mathrm{H}, \mathrm{Ar}-\mathrm{H}$ ), 8.59 (s, 1H, Ar-H), 8.0 (s, 1H, Ar-H), 7.98 (d, 1H, J7.9 Hz, Ar-H), 7.76 (t, 2H, J 8.3 Hz, Ar-H), 7.45 (dd, 1H, J 7.4 Hz, Ar-H), 7.32 (dd, $1 \mathrm{H}, J 7.6 \mathrm{~Hz}, \mathrm{Ar}-\mathrm{H}), 3.58$ (s, 2H, $\left.\mathrm{CH}_{2}\right), 2.38$ (s, $2 \mathrm{H}$,
$\left.\mathrm{CH}_{2}\right) ;{ }^{13} \mathrm{C}$ NMR $\left(100 \mathrm{MHz}, \mathrm{DMSO}-d_{6}\right) \delta 160.53,156.17$, $148.33,131.52,126.20,123.76,121.78,120.72,33.98$; EI-MS $\mathrm{m} / z[\mathrm{M}+\mathrm{H}]^{+} 441.32$; anal. calcd. for $\mathrm{C}_{19} \mathrm{H}_{14} \mathrm{BrN}_{5} \mathrm{O}_{3}: \mathrm{C}, 60.31$; H, 3.71; N, 18.48; found: C, 60.38; H, 3.78; N, 18.52 .

3-[5-(3-Bromophenyl)-1,3,4-oxadiazol-2-yl]- $N$-[2-oxo-1,2dihydro-3H-indol-3-ylidene]propane hydrazide (63)

Yield: $2.15 \mathrm{~g}$ (82\%); m.p. $175-177{ }^{\circ} \mathrm{C}, \mathrm{R}_{\mathrm{f}} 0.65$ (chloroform), $\lambda_{\max } 395 \mathrm{~nm}$, IR (KBr) $v / \mathrm{cm}^{-1} 3392,2816$, $1635,1605,1097 ;{ }^{1} \mathrm{H}$ NMR (DMSO) $\delta 8.71(\mathrm{~s}, 1 \mathrm{H}, \mathrm{Ar}-\mathrm{H})$, $8.61(\mathrm{~s}, 1 \mathrm{H}, \mathrm{Ar}-\mathrm{H}), 8.21(\mathrm{~s}, 1 \mathrm{H}, \mathrm{Ar}-\mathrm{H}), 7.88(\mathrm{~d}, 1 \mathrm{H}$, $J 7.9 \mathrm{~Hz}, \mathrm{Ar}-\mathrm{H}), 7.66$ (t, 2H, J 8.3 Hz, Ar-H), 7.42 (dd, 1H, $J 7.4 \mathrm{~Hz}, \mathrm{Ar}-\mathrm{H}), 7.22$ (dd, $1 \mathrm{H}, J 7.6 \mathrm{~Hz}, \mathrm{Ar}-\mathrm{H}), 3.20$ (s, 2H, $\left.\mathrm{CH}_{2}\right), 2.35$ (s, $\left.2 \mathrm{H}, \mathrm{CH}_{2}\right) ;{ }^{13} \mathrm{C}$ NMR (100 MHz, DMSO- $d_{6}$ ) $\delta$ 164.53, 155.17, 148.30, 130.52, 126.25, 125.78, 122.60, 118.74, 42.61, 33.98; EI-MS m/z [M + H] 441.09; anal. calcd. for $\mathrm{C}_{19} \mathrm{H}_{14} \mathrm{BrN}_{5} \mathrm{O}_{3}$ : C, 60.31; $\mathrm{H}, 3.71 ; \mathrm{N}, 18.48$; found: C, $60.33 ; \mathrm{H}, 3.75 ; \mathrm{N}, 18.51$.

\section{Pharmacology}

In vitro evaluation for anti-inflammatory activity by protein albumin denaturation method

All synthesized compounds were screened for anti-inflammatory activity by using in vitro method reported earlier by Mizushima and Kobayashi ${ }^{19}$ with slight modification. Accordingly, inhibition of albumin denaturation technique was studied, a $5.0 \mathrm{~mL}$ reaction mixture was prepared consisting of $0.2 \mathrm{~mL}$ of egg albumin (obtained from fresh hen's egg), $2.8 \mathrm{~mL}$ phosphate buffered saline ( $\mathrm{pH} 6.4$ ) and $2.0 \mathrm{~mL}$ of varying concentration of test compounds, so that final concentrations become 25, 50, 100 and $200 \mu \mathrm{g} \mathrm{mL} \mathrm{mL}^{-1}$. Similar volume of double distilled water served as control. Then, the mixtures were incubated at $37 \pm 2{ }^{\circ} \mathrm{C}$ in an incubator for $15 \mathrm{~min}$ and then heated at $70{ }^{\circ} \mathrm{C}$ for $5 \mathrm{~min}$. After cooling, their absorbance was measured at $660 \mathrm{~nm}$ by using vehicle as blank. Indomethacin with final concentration of 50 and $100 \mu \mathrm{g} \mathrm{mL}^{-1}$ was used as reference drug and treated similarly for determination of absorbance. The reading for this activity was taken on the same day and percent inhibition of protein denaturation was calculated by equation 1 , as follows:

\%Inhibition $=\frac{\left(\mathrm{Abs}_{\text {control }}-\mathrm{Abs}_{\text {sample }}\right)}{\mathrm{Abs}_{\text {control }}} \times 100$

In vivo evaluation

Anti-inflammatory activity

The anti-inflammatory activity was carried out according to the method of Halen et al. ${ }^{20}$ and employed 
with some modifications. ${ }^{18}$ Sprague Dawley rats were used for this study; seventeen groups with six rats per group were formed. All test samples were administered to animals at $100 \mathrm{mg} \mathrm{kg}^{-1}$ dosage, as suspension in $0.5 \%$ carboxymethyl cellulose in water. The samples were administered orally; after $60 \mathrm{~min}$ of drug dose, injection of $0.1 \mathrm{~mL}$ solution of carrageenan $\left(0.5 \mathrm{mg} 25 \mathrm{~mL}^{-1}\right)$ was injected into the sub-plantar tissue of the left hind paw of each rat. Out of this, one group was treated with standard drug indomethacin $\left(100 \mathrm{mg} \mathrm{kg}^{-1}\right)$. The initial volume of paw was measured within $30 \mathrm{~s}$ after carrageenan injection. Later on paw volume was measured after 1-5 h, respectively. The relative increase in the paw volume was calculated in the individual animal of the control, test, and standard groups, respectively. The percentage of inhibition of edema was calculated by the equation 2 , as follows:

Anti-inflammatory acitivity $(\%$ inhibition $)=\left[\left(1-\frac{D_{t}}{D_{c}}\right) \times 100\right]$

where $D_{t}$ means relative change in paw volume in test group and $D_{c}$ means relative change in paw volume in control group.

\section{Analgesic activity}

Analgesic activity test was performed following the method of Eddy and Leimbach. ${ }^{21}$ Analgesic activities of all synthesized compounds were quantified in vivo by Eddy's hot plate method using analgesiometer. Albino wistar mice were used for this study; seventeen groups with six rats per group were formed. All the test compounds were suspended in $0.5 \%$ of carboxymethylcellulose sodium (CMC) and administered orally. The albino wistar rats were treated with the newly synthesized derivatives (100 mg kg ${ }^{-1}$, p.o.) and standard drug indomethacin (100 $\mathrm{mg} \mathrm{kg}^{-1}$, p.o.). The animals were individually placed on the hot plate maintained at $55^{\circ} \mathrm{C}$, one hour after their respective treatments. The response time was noted as the time at which animals reacted to the pain stimulus either by paw licking or jump response. The relative increase in reaction time was measured at an interval of $0,30,60$ and $90 \mathrm{~min}$ in the individual animal of the control, test and the standard group (Table 1). The percent increase in reaction time was calculated using equation 3 as follows,

$\%$ Increase in reaction time $=\left[\frac{\left(I_{t}-I_{o}\right)}{I_{0}}\right] \times 100$

where $I_{t}$ is the reaction time at time $t$ and $I_{o}$ is the reaction time at time zero $(0 \mathrm{~min})$.

\section{Ulcerogenic activity}

Acute ulcerogenesis was done according to Cioli et al..$^{28}$ and modifications standardized in our laboratory. ${ }^{18}$ Albino rats (150-200 g) were divided into seven different groups

Table 1. Anti-inflammatory, analgesic, ulcerogenic index and molecular docking studies of synthesized compounds (49-63)

\begin{tabular}{|c|c|c|c|c|c|c|c|c|c|c|}
\hline \multirow{5}{*}{ Compound } & \multicolumn{5}{|c|}{ Anti-inflammatory activity } & \multirow{2}{*}{\multicolumn{3}{|c|}{ Analgesic activity }} & \multirow{5}{*}{$\begin{array}{l}\text { Ulcerogenic } \\
\text { index }\end{array}$} & \multirow{5}{*}{$\begin{array}{l}\text { Dock } \\
\text { score }\end{array}$} \\
\hline & \multicolumn{2}{|c|}{ In vitro } & \multicolumn{3}{|c|}{ In vivo } & & & & & \\
\hline & \multirow{3}{*}{ Absorbance $^{\mathrm{a}}$} & \multirow{3}{*}{$\begin{array}{c}\text { Inhibition of } \\
\text { denaturation / \% }\end{array}$} & \multicolumn{3}{|c|}{ Inhibition of paw edema ${ }^{\mathrm{b}} / \%$} & \multicolumn{3}{|c|}{ Increase in reaction / \% } & & \\
\hline & & & \multicolumn{3}{|c|}{ time $/ \mathrm{h}$} & \multicolumn{3}{|c|}{ time $/ \mathrm{min}$} & & \\
\hline & & & 1 & 2 & 3 & 30 & 60 & 90 & & \\
\hline 49 & 0.0401 & 61.64 & 11.6 & 13.7 & 13.9 & 19.45 & 66.81 & 71.98 & 0.24 & -3.91 \\
\hline 50 & 0.0409 & 66.59 & 35.8 & 40.9 & 42.7 & 35.86 & 77.11 & 84.11 & 0.35 & -4.44 \\
\hline 51 & 0.0407 & 66.19 & 38.4 & 43.1 & 45.5 & 21.86 & 76.14 & 83.17 & 0.56 & -4.37 \\
\hline 52 & 0.0403 & 65.58 & 13.4 & 14.1 & 14.9 & 11.92 & 70.35 & 81.11 & 0.52 & -3.45 \\
\hline 53 & 0.0402 & 65.07 & 30.3 & 30.5 & 30.6 & 24.55 & 29.72 & 35.00 & NT & -4.01 \\
\hline 54 & 0.0398 & 62.13 & 20.4 & 21.2 & 23.9 & 27.57 & 28.52 & 40.11 & NT & -3.33 \\
\hline 55 & 0.0403 & 65.63 & 20.2 & 21.4 & 21.7 & 26.69 & 23.91 & 26.69 & NT & -3.05 \\
\hline 56 & 0.0401 & 61.64 & 35.1 & 37.6 & 38.1 & 21.38 & 57.81 & 59.79 & NT & -3.27 \\
\hline 57 & 0.0402 & 63.18 & 35.5 & 40.0 & 40.5 & 44.91 & 51.12 & 54.29 & NT & -3.63 \\
\hline 58 & 0.0403 & 65.87 & 21.2 & 22.6 & 24.4 & 20.34 & 25.33 & 27.13 & NT & -1.85 \\
\hline 59 & 0.0397 & 61.58 & 33.34 & 35.56 & 36.12 & 27.16 & 29.12 & 32.13 & NT & -2.48 \\
\hline 60 & 0.0373 & 54.12 & 22.5 & 25.8 & 28.2 & 21.34 & 23.33 & 27.13 & NT & -0.16 \\
\hline 61 & 0.0403 & 65.12 & 23.1 & 25.2 & 33.9 & 11.96 & 58.35 & 70.11 & 0.39 & -2.20 \\
\hline 62 & 0.0404 & 64.16 & 28.5 & 31.8 & 38.2 & 21.34 & 23.38 & 25.13 & NT & -4.04 \\
\hline 63 & 0.0402 & 62.13 & 23.5 & 24.8 & 25.2 & 23.34 & 24.39 & 27.13 & NT & -4.46 \\
\hline Standard drug ${ }^{\mathrm{c}}$ & 0.0510 & 82.14 & 28.5 & 28.7 & 46.6 & 64.27 & 82.26 & 85.84 & 1.25 & -4.47 \\
\hline
\end{tabular}

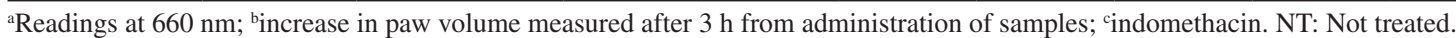


consisting of six animals in each one. Ulcerogenic activity was evaluated after p.o. administration of test compounds or indomethacin at the dose of $50 \mathrm{mg} \mathrm{kg}^{-1}$. Control rats received p.o. administration of vehicle (suspension of $1 \%$ methyl cellulose in water). Food but not water was removed $24 \mathrm{~h}$ before administration of the test compounds. After the drug treatment, the rats were fed normal diet for $17 \mathrm{~h}$ and then sacrificed. The stomach was removed and opened along the greater curvature, washed with distilled water and cleaned gently by dipping in saline. Gastric mucosa of the rats was examined by means of a four times binocular magnifier and the lesions were counted according to earlier reported method. ${ }^{18}$ Accordingly, the mean score of each treated group minus the mean score of the control group was considered to determine the severity index of gastric damage for compounds under study.

\section{Molecular docking study}

For docking purpose, the three-dimensional structure of COX-2 (protein data bank, PDB code 4Z0L) was obtained from RCSB PDB. ${ }^{29}$ The receptor molecule was refined and validated on the basis of Ramchandran plot using Biopredicta $\bigodot$ module on the Vlife MDS Molecular Modeling software, version 4.3.1.22 The Vlife MDS suit uses k-nearest neighbour genetic algorithm (KNN-GA) method for molecular docking. ${ }^{22}$ The scoring function is based on the basis of best suited moiety for the target with respect to energy and inter molecular interactions. The ligands that were already present within the receptor in bound form were removed to allow for docking protocol. All the ligands were prepared and docked for this study in flexible docking mode and atoms located within a range of $5.0 \AA$ from the amino acid residues were selected in the active site. Standard drug indomethacin was also included into the series of compounds under study to make comparison with respect to in silico analysis. The docking calculations and energy minimization were set in the Biopredicta $@$ module, most of the parameters were set default with 10000 cycles per molecule for the active site cavity No. 1 . Since this receptor is a homodimer nearly all amino acid residues are common in it, the active site cavity consisted of amino acid residues GLN374, LEU145, TRP139, TYR373, PRO127, ASN375, PRO538, SER541, GLY536, ASN537 and PHE142.

\section{Results and Discussion}

The reported investigation deals with synthesis and characterization of several hybrid derivatives from indole and 1,3,4-oxadiazole nucleuses linked via hydrazide chain to form final fifteen derivatives. To achieve these, two different steps were carried out to provide with substituted aryl acid hydrazides and indolidene hydrazinyl butanoic acids, which were further reacted to yield the final derivatives. In the first step, various substituted benzoic acids 1-15 esterifies in presence of ethanol and concentrated sulphuric acid to yield corresponding ethyl benzoates 16-30. These compounds were treated with hydrazine hydrate to yield the substituted aryl acid hydrazide 31-45. These compounds were not characterised on basis of NMR and mass analysis because they are reported in literature; hence, the physicochemical parameters were used and carry out the confirmation of these compounds. All the compounds 16-45 were analysed on the basis of melting point and TLC and found to be in understanding with the reported molecules.

In the second step, isatin ( $1 H$-indole-2,3-dione) $\mathbf{4 6}$ was reacted with hydrazine hydrate in presence of methanol under conditions of reflux to yield the 3-hydrazinylidene1,3-dihydro-2H-indol-2-one 47. NMR spectra of this compound exhibited prominent signals at $\delta 3.34 \mathrm{ppm}$ corresponding to the secondary amide proton, the aromatic protons belonging to fused benzene ring was exhibited around $\delta 7.0$ to $7.7 \mathrm{ppm}$ presenting four protons. The major spectral change was observed in the IR spectrum which provides with an appearance of primary amine functional group at $3411.12 \mathrm{~cm}^{-1}$. This structure was further confirmed on basis of mass spectral study which reflects the molecular weight of 162.24 on the ESI-MS in positive mode. In the next step, 47 was reacted with succinic anhydride under basic condition in an aprotic solvent such as dichloromethane to obtain 4-oxo-4-[2-(2-oxo-1,2dihydro-3H-indol-3-ylidene)-hydrazinyl] butanoic acid 48. This compound was confirmed on the basis of spectral studies; ${ }^{1} \mathrm{H}$ NMR exhibited singlets at $\delta 3.78$ and $2.23 \mathrm{ppm}$ for four protons belonging to two methylene carbons. The proton of hydroxyl group was observed at $\delta 12.44 \mathrm{ppm}$, whereas the protons of secondary amides were observed at $\delta 6.30 \mathrm{ppm}$. The four aromatic protons were reflected around $\delta 7.30$ to $8.0 \mathrm{ppm}$ for the benzene ring system. This was further confirmed from mass spectra in positive mode with molecular weight 262.02.

The final derivatives of this series 3-(5-substituted1,3,4-oxadiazol-2-yl)- $N$ '-[2-oxo-1,2-dihydro-3H-indol3-ylidene]propane hydrazides 49-63 were synthesized by carrying out cyclization of compounds 31-45 with 48 in presence of phosphorous oxychloride. These products were obtained in satisfactory yield and purity as studied on the thin layer chromatography and melting point studies. The structural confirmation was carried out on the basis of spectral studies, the IR spectra of these compounds exhibited absorbance for important functional groups, such as secondary amide at $3154 \mathrm{~cm}^{-1}$; the carbonyl group 
is indicated at $1655 \mathrm{~cm}^{-1}$ and the $\mathrm{C}=\mathrm{N}$ bond is reflected around $1618 \mathrm{~cm}^{-1}$. These groups are common to all the molecules from final derivatives. The ${ }^{1} \mathrm{H}$ NMR spectra of these compounds exhibited several characteristic NMR shifts. The ethylene protons were observed around $\delta 2.00$ to $3.85 \mathrm{ppm}$; the aromatic protons were observed within the range of $\delta 7.00$ to $8.50 \mathrm{ppm}$, representing the phenyl ring and the indole aromatic nucleus. Compound $\mathbf{5 0}$ presented with a hydroxyl proton at $\delta 9.49 \mathrm{ppm}$. These compounds were also analysed for their mass spectral characteristic and was found to be in good agreement with the results obtained for compound 50, which exhibited molecular mass of $[\mathrm{M}+\mathrm{H}]^{+} 378.11$ in the electron spray ionisation with positive mode of mass spectrometry.

All the synthesized compounds of the final series 49-63 were screened for in vitro anti-inflammatory activity based on assay developed by Mizushima and Kobayashi. ${ }^{19}$ This assay is also known as the protein albumin denaturation test as it involves the determination of percentage protection from denaturation of protein and thus its stabilization ultimately the inhibition of inflammation. The relativity of absorbance between test samples (compounds 49-63) with respect to control, designated the stabilization of protein and thus the inhibition of heat-induced protein (albumin) denaturation or the inhibition by derivatives and reference drug indomethacin. The increase in absorbance in the test compounds indicated better stabilization of proteins compared to standard drug indomethacin and the blank. Compounds $\mathbf{5 0}$ and $\mathbf{5 1}$ exhibited absorbance of 0.0409 and 0.0407 accounting for a 66.59 and $66.19 \%$ of denaturation, respectively. These are highest in this series of compounds followed by $65.87 \%$ (0.0403) for $\mathbf{5 8}$, then $65.63 \%$ (0.0403) for $\mathbf{5 5}$, and $65.07 \%$ (0.0402) for $\mathbf{5 3}$ (Table. 1). Other derivatives have also shown better percent of denaturation, which is in the range of 54.12 to $64.16 \%$; the standard drug indomethacin has exhibited percent of denaturation of $82.14 \%(0.0510)$. These results indicate that all the compounds have potential anti-inflammatory activity since the least active compound is also exhibiting a percent denaturation of $54.12 \%$, with more than fifty percent, whereas the most active compound presents inhibition of $66.59 \%$. In vitro assay displayed some features of this series with respect to substitutions; methyl, hydroxy, methoxy, chloro and nitro substituted derivatives, such as 50, 51, $\mathbf{5 8}$ and 55, possess good anti-inflammatory activity. Since these compounds were studied at various concentrations $\left(25,50,100\right.$ and $\left.200 \mu \mathrm{g} \mathrm{mL} \mathrm{m}^{-1}\right)$, it was decided to perform in vivo activity on all the derivatives to determine their antiinflammatory activity.

Compounds were evaluated for their in vivo antiinflammatory activity by carrageenan induced paw edema method. The protocol of animal experiments was approved by the Institutional Animal Ethics Committee (IAEC). The compounds were tested at $100 \mathrm{mg} \mathrm{kg}^{-1}$ oral dose and were compared with the standard drug indomethacin at $100 \mathrm{mg} \mathrm{kg}^{-1}$ oral dose. The tested compounds showed anti-inflammatory activity ranging from 13.9 to $45.5 \%$ after $3 \mathrm{~h}$ (Table 1). The anti-inflammatory results revealed that compounds 50, 51 and $\mathbf{5 8}$ exhibited good anti-inflammatory activity whereas compounds $\mathbf{5 3}$ and $\mathbf{5 5}$ showed moderate activity, and compounds 63, 62, 57, 54 and 49 showed low activities when compared with standard drug (indomethacin). These results illustrate that compounds substituted with methyl 51, hydroxy 50, methoxy 53 and nitro 55 at position 5 of the 1,3,4-oxadiazole ring system showed good anti-inflammatory activity, having maximum percentage of inhibition in edema. Surprisingly, the halogen derivatives such as chloro 54, fluoro 57, bromo 63 and acetyl 56 showed low activity. This indicates that the compound having $p$-substituted electron withdrawing groups may enhance anti-inflammatory activity and electron releasing groups diminished the activity. The activity declines with replacement of electronegative group by electropositive group. It is noteworthy that $\mathrm{NO}_{2}$ substitution of 3-nitrophenyl in $\mathbf{5 2}$ and 4-nitrophenyl in $\mathbf{5 5}$ induce remarkable change in the activity, i.e., compound substituted with 3-nitrophenyl $\mathbf{5 2}$ showed low activity while compound substituted with 4-nitrophenyl $\mathbf{5 5}$ showed good activity. So, $p$-substituted derivatives favour good activity than $m$-substituted derivatives.

The analgesic activity of the synthesized compounds was evaluated by hot plate test according to Eddy and Leimbach. ${ }^{21}$ The compounds were tested at $100 \mathrm{mg} \mathrm{kg}{ }^{-1}$ oral dose and were compared with the standard drug indomethacin at $100 \mathrm{mg} \mathrm{kg} \mathrm{kg}^{-1}$ oral dose. The tested compounds showed analgesic activity ranging from 25.13 to $84.11 \%$ (Table 1) after 90 min time cycle for the series of compounds under study. The analgesic screening results revealed that compounds 50, 51 and 52 showed good analgesic activity whereas compounds 49 and 61 showed moderate activity and compounds 56 and $\mathbf{5 7}$ showed low activity when compared with standard drug. The results illustrate that compounds substituted with 3-nitrophenyl $\mathbf{5 2}$ at position 5 of the 1,3,4-oxadiazole ring system showed good analgesic activity having elevated percentage increase in reaction time following the compounds $\mathbf{5 0}$ and $\mathbf{5 1}$ with maximum activity. In addition, compounds substituted with phenyl 49 and 2-fluorophenyl 61 showed moderate activities while compound substituted with 2,3-dimethoxy 53, 4-chlorophenyl 54 and 4-nitrophenyl 55 showed low activity. This indicates that the compounds having $p$-substituted and $m$-substituted electron withdrawing 
groups may enhance analgesic activity and electron releasing groups diminish the activity. From the above discussion, it is clear that compound substituted with 4-fluorophenyl $\mathbf{5 7}$ was found to be a fair anti-inflammatory agent with poor analgesic activity. These results suggest that unlike the substitution on indomethacin, the 1,3,4-oxadiazole ring system behaves differently and suggests for substitution with electron donating group (EDG) on the ring system.

The ulcerogenic activity was performed according to Cioli et al. ${ }^{28}$ and reports published earlier from our lab. ${ }^{18}$ Those compounds, which showed good results in antiinflammatory and analgesic activity, were screened for their ulcerogenic activity. The tested compounds exhibited activity in the range of 0.14 to 0.56 , whereas standard drug indomethacin presented very high severity index of 1.25 . Results of this activity indicate that compound $\mathbf{5 0}$ and 51 have a severity index of 0.35 and 0.56 , respectively. Compounds 49 and $\mathbf{6 1}$ exhibit severity index of 0.24 and 0.39 , respectively, which is the lowest compared to other tested compounds; these compounds lack good antiinflammatory activity. This experiment clearly indicates that $\mathbf{5 0}$ and $\mathbf{5 1}$ are better tolerated derivative of this series and are far safer than the standard drug indomethacin with respect to the ulcerogenic effect.

Indole is a very well-known nucleus and its derivative indomethacin is known to possess anti-inflammatory activity and used in therapy since last forty years. Accordingly, we proposed and derived several hybrids consisting of indole and oxadizole nuclei as inhibitor of the COX receptor, which is the target receptor for nearly all the NSAIDs. On the basis of in vitro and in vivo studies, it was found that compounds $\mathbf{5 0}$ and $\mathbf{5 1}$ exhibit good anti-inflammatory and analgesic activity. In order to investigate the molecular interactions and binding modes of some of the synthesized derivatives like 50, $\mathbf{5 1}, \mathbf{5 3}, \mathbf{5 5}$ and $\mathbf{6 2}$, we docked these derivatives with COX enzyme (PDB code 4Z0L) ${ }^{29}$ using licensed version of Vlife MDS 4.32 software tools. ${ }^{22}$ This COX-2 receptor was used for our study because of two reasons: first, this complex provides with a indole derivative, which can act as a reference molecule for docking; and the second reason was the similarity with our animal model. Prior to carrying out docking the COX-2 receptor was prepared for docking by removing the ligands in complex but the water molecules were retained for this particular study. The receptor was prepared by removing the already bound ligand and then repaired with for missing residues by Biopredicta ${ }^{\circ}$ tool. COX-2 is a homodimer and contains identical residues, active sites and channels; therefore, we employed a single chain for our study. This model was validated on the basis of
Ramchandran Plot analysis and was found to be satisfactory with the core and allowed count of about $98 \%$ including glycine and proline residues. This model was saved and further employed for carrying out docking studies.

The docking was carried out for all synthesized compounds. Figure 2 exhibits the docking mode of compound $\mathbf{5 0}$ and $\mathbf{5 1}$ with all three important interactions like hydrogen bonding, hydrophobic interaction and the Van der Waals forces interactions. Three compounds, 50, 51 and $\mathbf{5 3}$, have exhibited good activity with $\mathbf{5 0}$ and $\mathbf{5 1}$ being most active followed by the other. This was also observed with the results of molecular docking; the energies for $\mathbf{5 0}$ and 51 were found to be -4.44 and -4.37 , respectively, which is highest in the series of synthesized derivatives and comparable to standard drug indomethacin with score of -4.47 (Table 1). Compound $\mathbf{5 0}$ revealed that it binds to the active site of COX-2 receptor by forming hydrogen bond with GLY536 (bond length: $2.125 \AA$ ) and TYR373 (bond length: $2.325 \AA$; Figure 2a, 50). Hydrophobic interactions were found to be mostly between GLN374 with bond length of 4.635, 4.538 and 4.045, ARG376 (Figure 2b, 50). It also formed multiple Van der Waal interactions with the receptor amino acids having bond length between 2 to $3.9 \AA$ (Figure 2c, 50). Compound 51 prominently displayed that it binds to the active site of COX-2 receptor by forming two hydrogen bonds with ASN375 (bond length: 2.108 and 2.286 ̊̊; Figure 2a, 51). Hydrophobic interactions were found to be mostly among GLN374A, GLN374B, ASN375B, TYR373B, PRO127A, SER541B and ASN537B with bond length between 2.342 and 4.828 (Figure 2b, 51). It also formed multiple Van der Waal interactions with the receptor amino acids having bond length between 2.0 and $3.9 \AA$ (Figure 2c, 51).

The Van der Waal interactions were observed between the receptor and indole nucleus plus its substituted oxadiazole moiety. This molecule seemed to lay on both the chains as A and B of the homodimer, it is illustrated by fact that interactions were observed with GLY536A, PHE142B, TRP139B, PRO538A, ASN537A, GLN374A, ASN375A, ARG376B, ASN373B, GLY536B, TYR373B, PRO127A, PRO538B and ASN537B with bond length from 2.1 to $3.9 \AA$. Compounds $\mathbf{5 3}$ and $\mathbf{5 5}$ were found to show good dock score of -4.01 and -3.05 , respectively; these compounds also had interaction with TRP139B, PRO538A, ASN537A, GLN374A, ASN375A, ARG376B, ASN373B and ASN 537B, forming the hydrophobic and Van der Waals interactions. In case of compounds $\mathbf{6 2}$ and $\mathbf{6 3}$, the dock score was found to be comparatively high viz, -4.04 and -4.46 , respectively, but they did not show a good biological activity profile in wet lab studies. So, this dock score might be due to the presence of halogens, such 

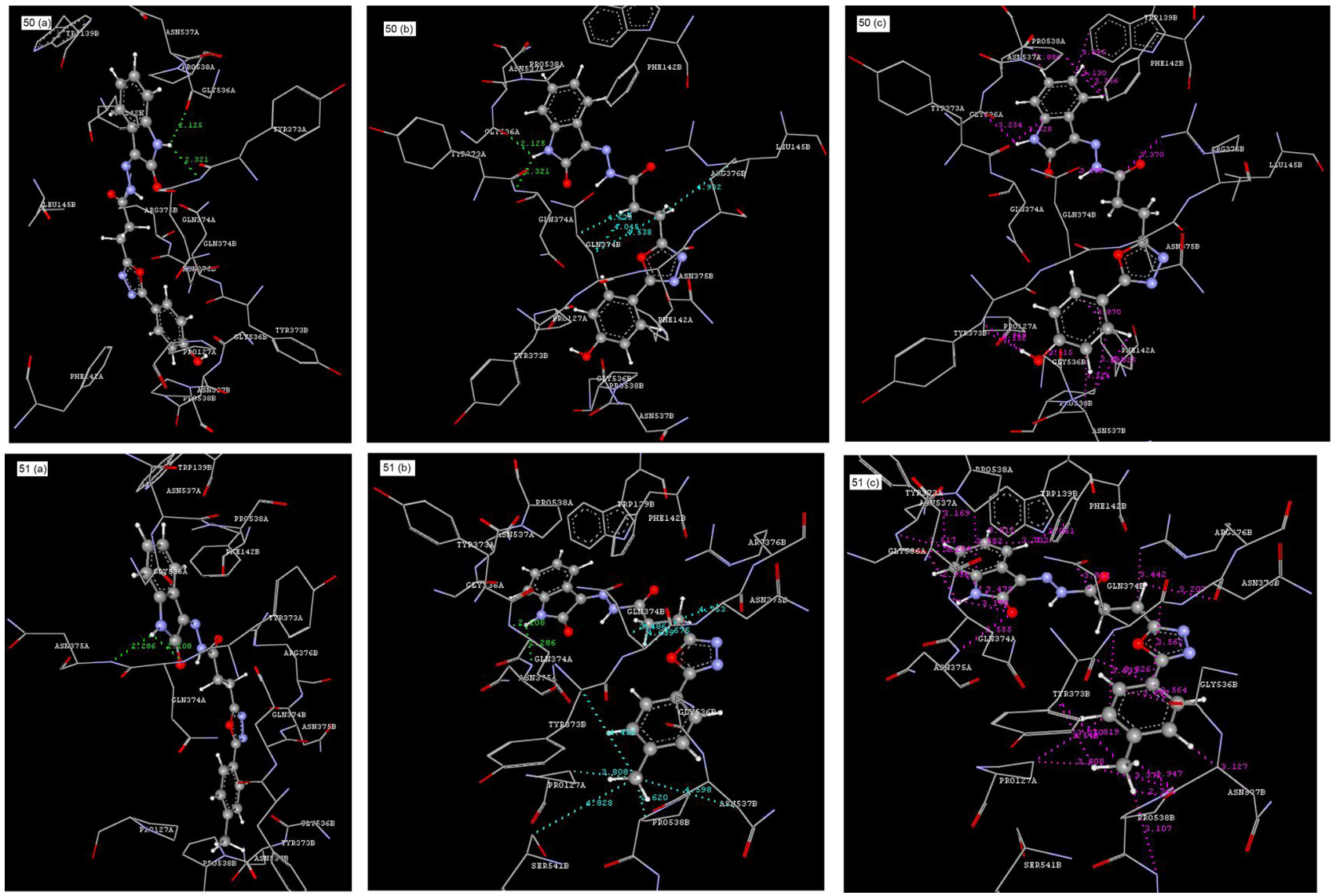

Figure 2. Molecular docking results for compound 50 (above) and 51 (below): (a) hydrogen bond interaction; (b) hydrophobic interaction; (c) Van der Waals interaction.

as the bromine. It is observed that presence of methyl or hydroxyl groups causes rise in activity as well as it can be seen that nitro substitution on the 1,3,4-oxadiazole causes increase in the activity. The docking of newly designed molecules has also presented their entry into deep sited region of the receptor, probably on the confluence of both the chains. Thus, it is considered that presence of indole and oxadiazole ring in the single molecule is beneficial for the activity, but the presence of halogen such as fluorine or chlorine on the 1,3,4-oxadiazole reduces the activity. Further, it is also noted that the position of substitution on the phenyl ring has great contribution towards the activity as an anti-inflammatory and analgesic compound.

In this preliminary communication, we have presented synthesis and pharmacological evaluation of novel $N^{\prime}$ (2-oxoindolin-3-ylidene)-3-(5-substituted phenyl-1,3,4oxadiazol-2-yl)propane hydrazide derivatives 49-63, obtained following a five step reactions. The reactions were simple and follow-up procedure resulted in pure compounds with satisfactory yield. In vitro and in vivo antiinflammatory activity revealed that compounds $\mathbf{5 0}$ and $\mathbf{5 1}$ exhibit comparable inhibition to standard drug indomethacin with added advantage of very less possibility of ulceration.
These compounds were found to be good analgesic agents also when compared with standard drug. These molecules exhibited that methyl and hydroxy substituted molecules are better tolerated as well as present better activity when compared to their chloro and fluoro counterparts. The molecular docking studies on these molecules also verified the wet lab results, compounds $\mathbf{5 0}$ and $\mathbf{5 1}$ exhibited hydrogen bonding, hydrophobic interactions as well as Van der Waals interactions. The dock scores and binding energy were found to be in good agreement with the pharmacological results. This work need to be further elaborated with respect to number of molecules, their versatility, animal models, and structure activity relationship studies and most importantly with respect to the $\mathrm{pK} / \mathrm{pD}$ studies.

\section{Conclusions}

In conclusion, we have described the design and synthesis of novel oxadiazolyl-2-oxoindolinylidene propane hydrazides as amide tethered hybrids of indole and oxadiazole and their evaluation for anti-inflammatory and analgesic activity. The compounds were successfully synthesised following five step reaction to yield fifteen 
derivatives as 3-(5-substituted-1,3,4-oxadiazol-2-yl)- $N^{\prime}$-[2oxo-1,2-dihydro-3H-indol-3-ylidene]propane hydrazides. In vitro and in vivo studies have exhibited final derivatives 50 and $\mathbf{5 1}$ as highly promising molecules with severity index of 0.35 and 0.56 , respectively, encouraging for an analgesic compound. The hydroxy and methyl substitutions on phenyl ring system provided with active compounds having percentage of inhibition of 84.11 and $83.17 \%$, respectively, compared to standard the drug at $85.84 \%$. Molecular docking studies are also in agreement with the pharmacological evaluation with potent compounds exhibiting dock score of -4.44 . It can be stated that these compounds can be further studied for their structureactivity relationship (SAR) studies and developed into potential lead molecules.

\section{Supplementary Information}

Supplementary data are available free of charge at http://jbcs.sbq.org.br as PDF file.

\section{Acknowledgments}

The author D. K. is thankful to Principal, Kamla Nehru College of Pharmacy for providing the support to carry out this research work.

\section{References}

1. Arkin, M. R.; Tang, Y.; Wells, J.; Chem Biol. 2014, 21, 1102.

2. Gediya, L. K.; Njar, V. C.; Expert. Opin. Drug Discovery 2009 , 4, 1099.

3. Haubrich, D. R.; Ward, S. J.; Baizman, E.; Bell, M. R.; Bradford, J.; Ferrari, R.; Miller, M.; Perrone, M.; Pierson, A. K.; Saelens, J. K.; J. Pharmacol. Exp. Ther. 1990, 255, 511.

4. Solomon, S. D.; McMurray, J. J.; Pfeffer, M. A.; Wittes, J.; Fowler, R.; Finn, P.; Anderson, W. F.; Zauber, A.; Hawk, E.; Bertagnolli, M.; N. Engl. J. Med. 2005, 352, 1071.

5. Krishnan, S. S.; Atmakuru, R.; Biol. Pharm. Bull. 2001, 24, 1149.

6. Chaluvaraju, K. C.; Zaranappa, S. J.; Res. J. Pharm. Biol. Chem. Sci. 2011, 2, 541 .

7. Pandeya, S. N.; Sriram, D.; Acta Pharm. Turc. 1998, 40, 33.

8. Kucukguzel, S. G.; Mazi, A.; Sahin, F.; Ozturk, S.; Stables, J.; Eur. J. Med. Chem. 2003, 38, 1005.

9. Loncle, C.; Brunel, J. M.; Vidal, N.; Dherbomez, M.; LetourNeux, Y.; Eur. J. Med. Chem. 2004, 39, 1067.
10. Varma, R. S.; Nobles, W. L.; J. Med. Chem. 1967, 10, 972.

11. Holla, B. S.; Rao, S. B.; Shridhara, K.; Akberali, P. M.; Il Farmaco 2000, 55, 338.

12. Gaston, M. A.; Dias, L. R. S.; Freitas, A. C. C.; Miranda, A. L. P.; Barreiro, E. J.; Pharmac. Acta Helvet. 1996, 71, 213.

13. Adityakumar, K.; Suroor, A. K.; Mohammad, A.; Asif, H. H.; Mymoona, A.; Acta Pol. Pharm. 2011, 68, 381.

14. Srivastava, A.; Pandeya, S. N.; Int. J. Pharm. Sci. Rev. Res. 2010, 1,115 .

15. Panneerselvam, P.; Reddy, P. S.; Murali, K.; Kumar, N. R.; Pharm. Chem. 2010, 2, 28.

16. Wagle, S.; Vasudeva, A. A.; Suchetha, N. K.; Indian J. Chem. 2008, 47B, 439.

17. Jayashankar, B.; Rai, K. M. L.; Baskaran, N.; Sathish, H. S.; Eur. J. Med. Chem. 2009, 44, 3898.

18. Chikhale, R.; Bhole, R.; Khedekar, P.; Bhusari, K.; Eur. J. Med. Chem. 2009, 44, 3645.

19. Mizushima, Y.; Kobayashi, M.; J. Pharm. Pharmacol. 1968, $20,169$.

20. Halen, P. K.; Chagti, K. K.; Giridhar, R.; Yadav, M. R.; Chem. Biol. Drug. Des. 2007, 70, 450.

21. Eddy, N. B.; Leimbach, D.; J. Pharmacol. Exp. Ther. 1953, 107, 385.

22. Chikhale, R.; Menghani, S.; Babu, R.; Bansode, R.; Bhargavi, G.; Karodia, N.; Rajasekharan, M. V.; Paradkar, A.; Khedekar, P.; Eur. J. Med. Chem. 2015, 96, 30.

23. Chikhale, R.; Thorat, S.; Pant, A.; Jadhav, A.; Thatimala, K. C.; Bansode, R.; Bhargavi, G.; Karodia, N.; Rajasekharan, M. V.; Paradkar, A.; Khedekar, P.; Bioorg. Med. Chem. 2015, 23, 6689.

24. Furniss, B.; Hannaford, A. H.; Smith, P. W. G.; Tatchell, A. R.; Vogel's Textbook of Practical Organic Chemistry, $5^{\text {th }}$ ed.; Longman: Harlow, 1998.

25. Hassan, M.; Kadi, A. A.; Aziz, A. A.; US pat. 8,497,296 B2 2012 (CA 485763-70-0).

26. Sun, A.; Shoji, M.; Lu, Y.; Liotta, J. D. C.; Snyder, J. P.; J. Med. Chem. 2006, 49, 3153.

27. Husain, A.; Ahmad, A.; Alam, M. M.; Ajmal, M.; Ahuja, P.; Eur. J. Med. Chem. 2009, 44, 3798.

28. Cioli, V.; Putzolu, S.; Rossi, V.; Barcellona, P. S.; Corradino, C.; Toxicol. Appl. Pharmacol. 1979, 50, 283.

29. Neumann, W.; Xu, S.; Sarosi, M. B.; Scholz, M. S.; Crews, B. C.; Ghebreselasie, K.; Banerjee, S.; Marnett, L.; Hey-Hawkins, H. C.; ChemMedChem 2016, 11, 175.

Submitted: January 15, 2016 Published online: March 22, 2016 